\title{
Helminthostachys zeylanica alleviates hepatic steatosis and insulin resistance in diet-induced obese mice
}

\author{
Ting-Chen Chang ${ }^{1}$, Hao Chiang ${ }^{2}$, Yu-Heng Lai ${ }^{3}$, Yu-Ling Huang ${ }^{4,5}$, Hsiu-Chen Huang ${ }^{6}$, Yu-Chih Liang ${ }^{1,7}$, \\ Hui-Kang Liu ${ }^{4,8}$ and Cheng Huang ${ }^{1,2,9^{*}}$
}

\begin{abstract}
Background: Obesity and its associated health conditions, type 2 diabetes mellitus (T2DM) and nonalcoholic fatty liver disease (NAFLD), are worldwide health problems. It has been shown that insulin resistance is associated with increased hepatic lipid and causes hepatic steatosis through a myriad of mechanisms, including inflammatory signaling.

Methods: Helminthostachys zeylanica $(\mathrm{HZ})$ is used widely as a common herbal medicine to relieve fever symptoms and inflammatory diseases in Asia. In the present study, we evaluated whether $\mathrm{HZ}$ has therapeutic effects on obesity, NAFLD and insulin resistance. The protective effects of $\mathrm{HZ}$ extract were examined using free fatty acidinduced steatosis in human HuS-E/2 cells and a high-fat diet-induced NAFLD in mice.

Results: The major components of the HZ extract are ugonins J and K, confirmed by HPLC. Incubation of human hepatocytes, HuS-E/2 cells, with palmitate markedly increased lipid accumulation and treatment with the HZ extract significantly decreased lipid deposition and facilitated AMPK and ACC activation. After 12 weeks of a high-fat diet with $\mathrm{HZ}$ extract treatment, the HFD mice were protected from hyperlipidemia and hyperglycemia. $\mathrm{HZ}$ extract prevented body weight gain, adipose tissue expansion and adipocyte hypertrophy in the HFD mice. In addition, fat accumulation was reduced in mice livers. Moreover, the insulin sensitivity-associated index, which evaluates insulin function, was also significantly restored.
\end{abstract}

Conclusions: These results suggest that $\mathrm{HZ}$ has a promising pharmacological effect on high-fat diet-induced obesity, hepatic steatosis and insulin resistance, which may have the potential for clinical application.

Keywords: Helminthostachys zeylanica, Hepatic steatosis, Insulin resistance, Obesity

\section{Background}

Obesity, fatty liver and dysregulated insulin action are strongly associated and are currently a worldwide health problem [1]. Fatty liver is the initial stage of nonalcoholic fatty liver disease (NAFLD), which is caused by an imbalance of lipid metabolism and is a common metabolic symptom [2]. NAFLD and type 2 diabetes mellitus (T2DM) frequently coexist because of sharing the similar

\footnotetext{
* Correspondence: chengh@ym.edu.tw

${ }^{1}$ Ph.D. Program in Medical Biotechnology, College of Medical Science and Technology, Taipei Medical University, Taipei 11001, Taiwan

${ }^{2}$ Department of Biotechnology and Laboratory Science in Medicine, National Yang-Ming University, No. 155, Sec. 2, Linong St., Beitou District, Taipei 11221, Taiwan

Full list of author information is available at the end of the article
}

pathogenic features of excess adiposity and insulin resistance [3]. Dysregulation of hepatic lipid homeostasis is thought to be important in the development of fatty liver, such as reduced fatty acid oxidation, enhanced de novo lipogenesis, elevated hepatic fatty acid influx, and/ or increased systemic insulin resistance [4]. Although the mechanisms involved in lipid-induced insulin resistance are not fully understood, it is well-known that nonalcoholic fatty liver disease (NAFLD), which causes nonalcoholic steatohepatitis (NASH), is one of the most relevant factors that leads to metabolic diseases and insulin resistance [5]. Indeed, therapies for fatty liver disease are aimed at reducing body weight and improving insulin sensitivity to alleviate the associated metabolic

(c) The Author(s). 2019 Open Access This article is distributed under the terms of the Creative Commons Attribution 4.0 International License (http://creativecommons.org/licenses/by/4.0/), which permits unrestricted use, distribution, and reproduction in any medium, provided you give appropriate credit to the original author(s) and the source, provide a link to the Creative Commons license, and indicate if changes were made. The Creative Commons Public Domain Dedication waiver (http://creativecommons.org/publicdomain/zero/1.0/) applies to the data made available in this article, unless otherwise stated. 
syndrome [6, 7]. Now, novel therapeutic strategies for NAFLD progression have stimulated great interest in terms of developing effective treatments for lipidassociated metabolic disorders [8].

Helminthostachys zeylanica (L.) Hook. (HZ), the only fern-like plant of the Ophioglossaceae, is distributed widely in Southeast Asia and has been used as a folk medicine for centuries [9]. It has been shown that $\mathrm{HZ}$ contains prenylated flavonoids and quercetin, which have inhibitory effects on human neutrophils [10]. In addition, the main components in HZ, flavonoids, have antioxidant and anti-inflammatory activities [10, 11]. Previous studies have shown that one of the main compounds in $\mathrm{HZ}$, ugonin $\mathrm{K}$, promotes osteogenesis through the Src-associated pathway and activates downstream Runx2 and oxterix [12]. Furthermore, HZ extract was considered to have neuroprotective activity because of its anti-inflammatory activity on human astrocytes, through bradykinin-induced MMP-9 signaling [13]. Another bioactive compound extracted from HZ, ugonin J, is considered to be a potential inhibitor of cell migration and neointima formation through MMP-2 and -9 pathways [14]. Rhizomes of $\mathrm{HZ}$ have been used for variety of purposes, including protection against liver damage [15]. However, the therapeutic effect of $\mathrm{HZ}$ on abnormalities of lipid and glucose metabolism remains unclear.

Previously, we established a human fatty liver cell model, based on HuS-E/2 immortalized human primary hepatocytes [16], and made use of a mouse model of metabolic syndrome with high-fat diet (HFD), which showed significant dyslipidemia and insulin resistance, and expressed hepatic steatosis markers [17]. Because of the vicious circle between NAFLD and insulin resistance, in this study, we applied our optimized human fatty liver cell model and HFD mouse model of metabolic disorder and investigated the potential restorative therapeutic effects of $\mathrm{HZ}$.

\section{Methods}

\section{H. zeylanica $(\mathrm{HZ})$ extract preparation}

Rhizomes of $\mathrm{HZ}$ were purchased from the Wanhua herbal market (Taipei, Taiwan) and identified by comparison with the voucher specimen (NRICM-99-003), which is already deposited at the herbarium of the National Research Institute of Chinese Medicine, Taiwan. HZ rhizomes $(531 \mathrm{~g})$ were heated and extracted with $2.5 \mathrm{l}$ of $\mathrm{EtOH}-\mathrm{H}_{2} \mathrm{O}$ (1:1) under reflux for $1 \mathrm{~h}$. The filtrate was concentrated and lyophilized to yield HZ extract (29 g, yield $5.46 \%)$.

\section{Purification of ugonin $\mathrm{J}$ and ugonin $\mathrm{K}$}

The preparation of ugonins $J$ and ugonin $\mathrm{K}$ were prepared as described previously [11]. Briefly, the rhizomes of $\mathrm{HZ}(12 \mathrm{~kg})$ were extracted with $\mathrm{EtOH}(201 \times 3)$ at
$50{ }^{\circ} \mathrm{C}$ for $24 \mathrm{~h}$. The concentrated EtOH extract $(460 \mathrm{~g})$ was partitioned between EtOAc and $\mathrm{H} 2 \mathrm{O}$, and the EtOAc extract (153 g) was applied to a silica gel column eluted with gradient solvent systems of $n$-hexane-EtOAc (20:1-1:10) and EtOAc-MeOH (10:1-1:1) to yield 16 fractions (Fr-1-Fr-16). Fraction Fr-7, the eluate of $n$ hexane-EtOAc $=1: 2$, was further subjected to a silica gel $\mathrm{CC} \quad(\mathrm{CH} 2 \mathrm{Cl} 2-\mathrm{MeOH}=30: 1)$ and Sephadex $\mathrm{LH}-20$ $(\mathrm{MeOH}-\mathrm{H} 2 \mathrm{O}=5: 1)$ to give ugonin $\mathrm{J}(26.3 \mathrm{mg})$ and ugonin K (18.6 mg), respectively.

\section{Reverse-phase HPLC chemical fingerprint analysis of $\mathrm{HZ}$ extract}

The HZ extract $(1.0 \mathrm{~g})$ was refluxed in $20 \mathrm{ml}$ methanol for $30 \mathrm{~min}$ and filtered. The filtrate volume was then adjusted to $50 \mathrm{ml}$ with the same solvent. A $10 \mu \mathrm{l}$ portion of the solution was injected into the HPLC system, an Agilent 1100 series equipped with a G1311A Quat Pump, a G1379A degasser, a G1315B photodiode array detector, a 1200 series G1329A autosampler, and a column oven $\mathrm{H}$ 650 (Chrom Tech, TNC.). A Cosmosil 5C18-AR-II column was utilized with a mobile phase of $\mathrm{MeOH}-\mathrm{H}_{2} \mathrm{O}$ ( $0.1 \%$ phosphoric acid, v/v) using a linear gradient, which started from $70 \% \mathrm{MeOH}$ for $35 \mathrm{~min}$, increasing to $75 \%$ in $10 \mathrm{~min}$, and finally reaching $100 \%$ at $65 \mathrm{~min}$ with flow rate of $1.0 \mathrm{ml} / \mathrm{min}$. The column oven was set at $30^{\circ} \mathrm{C}$ and the UV detection wavelength was set at $344 \mathrm{~nm}$.

\section{Antibodies, reagents and Western blot analysis}

Palmitate, Oil red $\mathrm{O}$, and luteolin were purchased from Sigma-Aldrich, St. Louis, MO. Antibodies against AMPK, pACC (Ser 79), ACC, SREBP-1c, CPT1, and tubulin were from Genetex. The anti-pAMPK (Thr 172) antibodies were obtained from Millipore, and the HRPconjugated anti-mouse or anti-rabbit secondary antibodies were from Jackson ImmunoResearch Laboratories Inc. Western blot analysis was performed as described previously [16].

\section{Cell culture and oil red $O$ staining}

HuS-E/2 cells were kindly provided by Dr. Shimotohno (Kyoto University, Japan) and maintained as described previously [18]. The lyophilized HZ extract was solubilized in DMSO as stock at the concentration of $25 \mathrm{mM}$ and diluted to the indicated concentration. DMSO was used as the vehicle for experimental control. For the fatty liver disease cell model, HuS-E/2 cells were cultured with $0.1 \mathrm{mM}$ palmitate for $18 \mathrm{~h}$. To measure lipid content in HuS-E/2 cells, the oil red O method was used as previously described [16].

\section{Quantitative real-time polymerase chain reaction}

For messenger RNA (mRNA) analysis, real-time polymerase chain reaction (RT-PCR) was performed as 
described previously [19]. The primer sets used in this study are listed in Additional file 1: Table S1.

\section{Animals}

4-week-old male C57BL/6 J mice were obtained from BioLASCO Taiwan Co, Ltd., Taiwan. All mice were housed under constant temperature $\left(24{ }^{\circ} \mathrm{C}\right)$ with a $12 \mathrm{~h}$ light/dark cycle at the Animal Center of the National Research Institute of Chinese Medicine, Taipei, Taiwan. Mice fed with a standard diet and adapted to the environment for one week were subsequently divided randomly into three groups and fed a normal diet (ND group, $n=10$ ), HFD (HFD group, $\mathrm{n}=10,30 \%$ fat and $1 \%$ cholesterol), or HFD with $0.5 \% \mathrm{HZ}$ extract (HFD-HZ group, $\mathrm{n}=10$ ) for 12 weeks. The estimated daily intake amount of $\mathrm{HZ}$ extract is about $578 \mathrm{mg} / \mathrm{kg} /$ day. On the day of sacrifice, a laparotomy was performed under ketamine and xylazine anesthesia (intramuscular injection of $100 \mathrm{mg} / \mathrm{kg}$ body mass and $5 \mathrm{mg} / \mathrm{kg}$ body mass, respectively), and mice were sacrificed via cardiac-puncture (to collect blood for the final time point) followed by cervical dislocation. The liver and adipose tissue were removed, rinsed with physiological saline, weighed, immediately frozen in liquid nitrogen, and stored until analysis. The animals used in this research were approved by the Animal Research Committee of the NRICM (IACUC no. 105-520-2). All experimental procedures were followed The Guide for the Care and Use of Laboratory Animals (NIH publication, 85-23, revised 1996) and the guidelines of the Animal Welfare Act, Taiwan.

\section{Biochemical characterization of plasma and histological analysis of fat and liver tissues}

The plasma, epididymis adipose, and liver tissues were collected from each sacrificed mouse. The biochemical analysis of plasma and histological analysis of the fat and liver tissues were performed as described previously [20].

Blood glucose, plasma insulin and the homeostasis model assessment of insulin resistance index (HOMA-IR)

The $12 \mathrm{~h}$ fasting blood glucose was measured with a glucose analyzer (EASYTOUCH, Taiwan). The plasma insulin and HOMA-IR were detected and calculated as described previously [20].

\section{Statistical analysis}

All data are expressed as the mean \pm SD from for three separate experiments. More than two sets of data were accessed by one-way ANOVA with Dunnett's multiple comparison test. The values significantly different from the control were indicated by asterisks (*, $p<0.05$; **, $p<0.01$; ***;,$p<0.001$.).

\section{Results}

Identification of major components in $\mathrm{H}$. zeylanica $(\mathrm{HZ})$ extract

Rhizomes of HZ were extracted and the chemical components were analyzed. HPLC analysis was performed on the $\mathrm{HZ}$ extract and two of the individual ingredients, ugonins $\mathrm{J}$ and $\mathrm{K}$ were isolated [11] and used as standard markers for quality control of $\mathrm{HZ}$ material. Both standard markers were well separated and their purities were determined by HPLC to be more than $98 \%$. The HPLC chromatogram of the $\mathrm{HZ}$ extract showed two major peaks at 44.484 and $60.466 \mathrm{~min}$. (Fig. 1a), corresponding to ugonin J (44.588 min.) (Fig. 1b) and ugonin K (60.276 min.) (Fig. 1c) under the same conditions.

\section{The effect of $\mathrm{HZ}$ on palmitate-induced cellular lipid accumulation in a human fatty liver cell model}

Fatty liver disease is mainly attributable to triglyceride accumulation in the hepatocytes [21]. To determine the effect of $\mathrm{HZ}$ extract on esterification in human liver cells and deposition of fatty acid as lipid droplets, HuS-E/2 immortalized human primary hepatocytes were used as a human fatty liver cell model [16]. HuS-E/2 cells were incubated with palmitate and $100 \mu \mathrm{g} / \mathrm{ml}$ of $\mathrm{HZ}$ extract for $18 \mathrm{~h}$. The lipid content of the cells was observed by Oil-Red O staining and quantified. As shown in Fig. 2a, compared to HuS-E/2 cells with only palmitate, cells incubated with the HZ extract showed significantly less lipid accumulation. The reduction of cellular lipid accumulation to $39 \%$ with the treatment with $\mathrm{HZ}$ extract was confirmed by image quantification (Fig. 2b). Because we found HZ extract had an inhibitory effect on lipid deposition in human hepatocytes, possible molecular mechanisms were explored. AMP-activated protein kinase (AMPK) was reported to regulate fat metabolism in the liver and change with cellular energy status [22]. To determine whether HZ extract increased levels of AMPK and its activation, HuS-E/ 2 cells were incubated with $0.1 \mathrm{mM}$ palmitate in the presence or absence of $\mathrm{HZ}$ extract and the expression of AMPK was assessed by western blotting. Levels of phospho-AMPK (pAMPK) at Thr-172 were assessed to evaluate AMPK activation. Ugonins $\mathrm{J}$ and $\mathrm{K}$ are structurally related to natural flavonoid luteolin [23], which has been demonstrated to attenuate hepatic steatosis [24]. Therefore, the agent luteolin was used as a positive control drug in the subsequent experiment. As shown in Fig. 2c, HZ extract increased AMPK phosphorylation at Thr-172 in palmitatetreated $\mathrm{HuS}-\mathrm{E} / 2$ cells. In addition, the activation of AMPK's downstream target enzyme, acetyl-CoA carboxylase (ACC), by phosphorylation at Ser-79 was also measured. HZ extract significantly increased ACC protein phosphorylation. The results indicated that $\mathrm{HZ}$ extract facilitated AMPK and ACC activation in HuS-E/2 


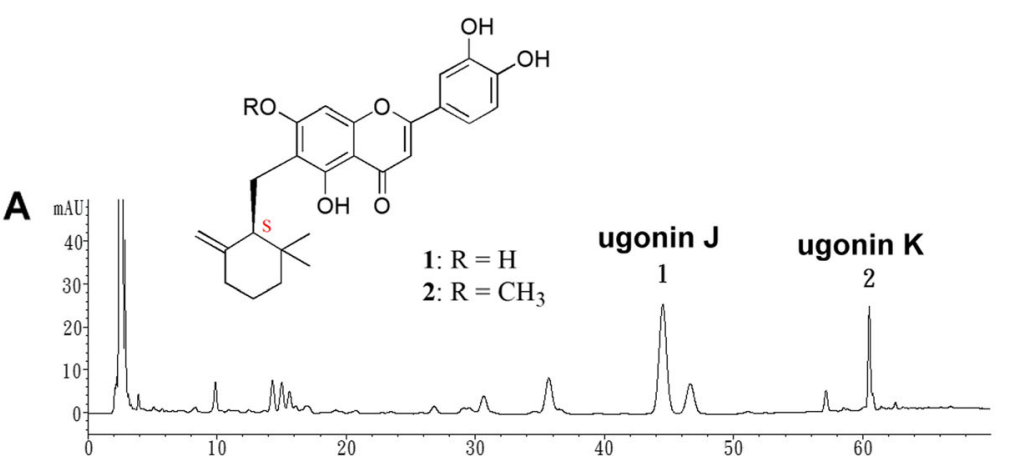

HZ extract

B

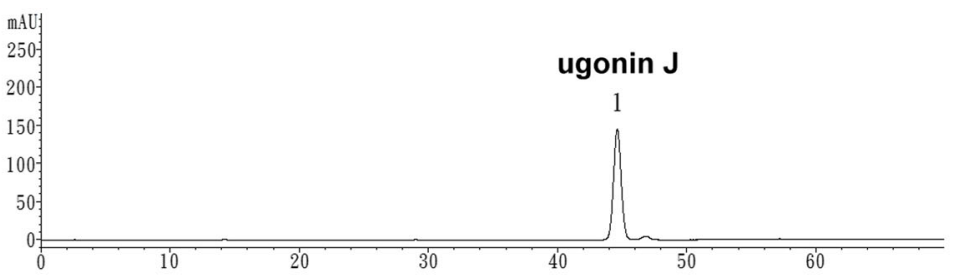

Standard

C

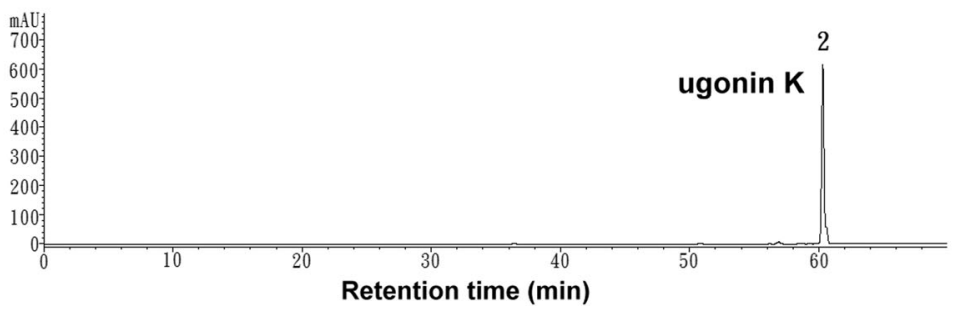

Standard

Fig. 1 Characterization of HZ extract. a HPLC chromatograms of HZ extract. Two major peaks were identified in the HZ extract. $\mathbf{b}$ Ugonin J and (c) ugonin $\mathrm{K}$ were used as standards. The chemical structures of the ugonins are shown

cells under high-fat conditions. HZ extract showed a stronger effect on AMPK and ACC activation than luteolin in HuS-E/2 cells.

We also identified the changes in proteins associated with fatty acid synthesis and $\beta$ oxidation. Compared to the palmitate group, $\mathrm{HZ}$ extract substantially decreased sterol regulatory element-binding transcription factor $1 \mathrm{c}$ (SREBP-1c) protein expression, which is involved in fatty acid synthesis (Fig. 2d). Carnitine palmitoyltransferase I (CPT1) functions as catalyzing fatty acids by $\beta$ oxidation [25]. Treatment with HZ extract greatly increased CPT1 protein expression, compared to the palmitate group. We determined whether lipid metabolism-related genes in the hepatocytes were influenced by HZ intervention. The expression of transcription factors, peroxisome proliferator-activated receptor alpha (PPAR $\alpha)$ and peroxisome proliferator-activated receptor delta (PPAR $\delta$ ), associated with fatty acid $\beta$ oxidation were markedly increased by treatment with HZ extract, compared to the palmitate and luteolin groups (Fig. 2e). The activities of genes involved in de novo lipogenesis in the hepatocytes, SREBP-1c and peroxisome proliferator-activated receptor gamma (PPARY) were substantially higher in the palmitate group than the non-treated group, while all the genes were expressed at greatly lower levels after treatment with $H Z$ extract and luteolin, compared with the palmitate group (Fig. 2f). Taken together, the results suggest $\mathrm{HZ}$ extract had a better effect than luteolin on inhibition of fatty acid synthesis and activation of fatty acid $\beta$ oxidation in palmitate-treated $\mathrm{HuS}-\mathrm{E} / 2$ hepatocytes.

\section{$\mathrm{HZ}$ lowered the body weight and food efficiency ratio of HFD mice}

The effect of $\mathrm{HZ}$ extract on lipemia syndrome and fatty liver was examined in an HFD mouse model. Five-weekold male C57BL/6 J mice were fed with normal diet (ND group, $n=10$ ), HFD (HFD group, $\mathrm{n}=10$ ), or HFD along with $0.5 \%$ lyophilized HZ extract (HFD-HZ group, $n=10)$ for 12 weeks. The morphology of the ND, HFD, and HFD-HZ mice was observed, as shown in Fig. 3a. The size and waist were obviously smaller in the ND group and HFD-HZ group than the HFD mice. The weight of HFD-HZ mice was significantly lower than the HFD mice after 12 weeks of diet supplemented with HZ (Fig. 3b). The food efficiency ratio (FER) was much lower in the HFD-HZ group than the HFD group (Fig. 3d), although the amount of food consumed did not differ significantly (Fig. 3c). 
A

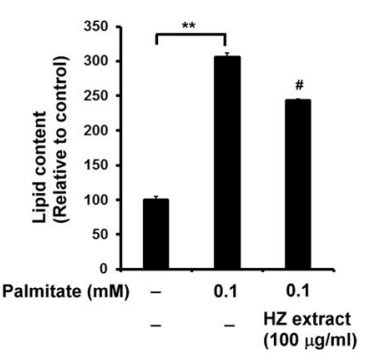

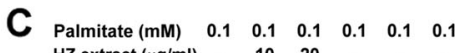
$\begin{array}{llccccc}\mathrm{HZ} \text { extract }(\mu \mathrm{g} / \mathrm{ml}) & - & 10 & 20 & - & - & - \\ \text { Luteolin }(\mu \mathrm{g} / \mathrm{ml}) & - & - & - & - & 10 & 20\end{array}$

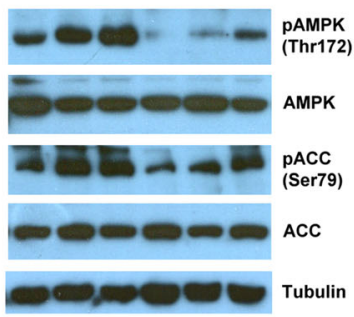

D

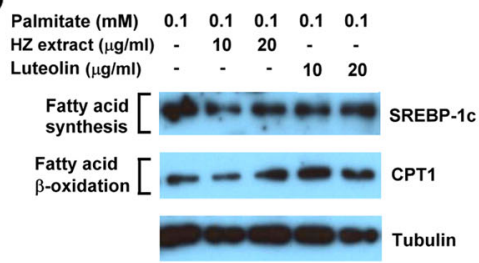

B

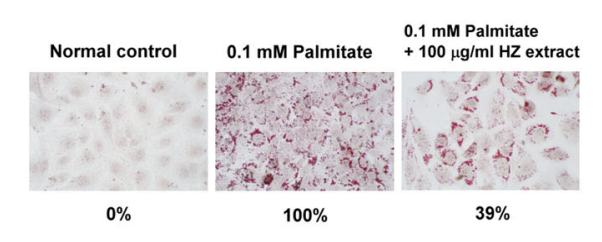

$100 \%$

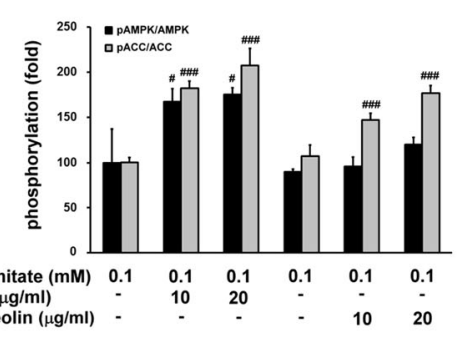

E

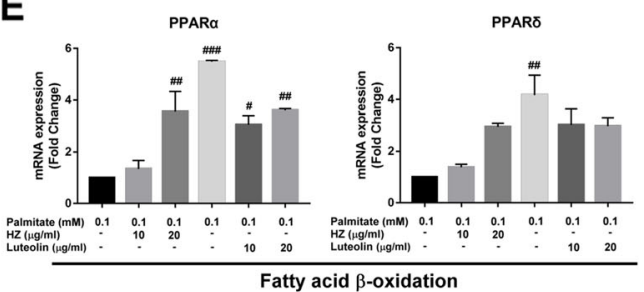

$\mathbf{F}$

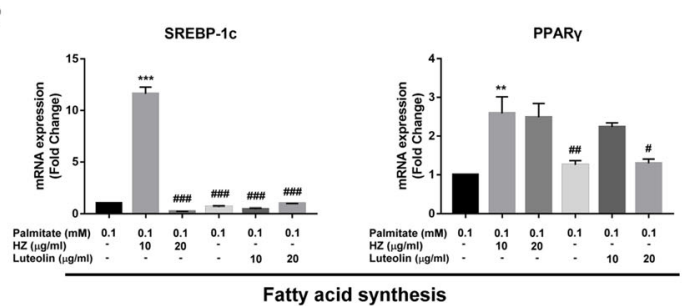

Fig. 2 Inhibition by HZ extract of palmitate-induced lipid accumulation in a human fatty liver cell model. a Quantitative analysis of lipid deposition in the Oil-Red O stained HuS-E/2 cells. b Images of the Oil-Red O stained HuS-E/2 cells were captured using a microscope at 400X original magnification. $\mathbf{c}$ Western blotting for phosphorylation of AMPK at Thr172 and ACC at Ser-79, total AMPK, ACC and tubulin. d Western blotting for SREBP-1C, CPT1, and tubulin. Tubulin served as a loading control. Quantitative analysis with Multi Gauge V3.0 is shown. e The levels of fatty acid $\beta$ oxidation-related genes, PPARa and PPARS. $\mathbf{f}$ The levels of fatty acid synthesis-related genes, SREBP-1c and PPARY. The data represent the mean \pm SEM for three independent experiments. ND vs. palmitate: ${ }^{*} p<0.05$; ${ }^{* *} p<0.01 ;{ }^{* * *} p<0.001$. palmitate vs. HZ extract: \#p $<0.05$; $\# \# p<0.01 ; \# \# p<0.001$

This suggests that HZ extract causes reduced food uptake, which may be the reason the mice gain less weight.

\section{$\mathrm{HZ}$ decreased fat deposition in adipocytes}

A feature of obesity is increasing lipid accumulation within adipocytes, leading to excessive visceral fat deposits. Therefore, the epididymis adipose tissue (EAT) was dissected and measured after 12 weeks of experimental diet. The mass of EAT in the HFD group was significantly higher than the ND and HFD-HZ groups (Fig. 4a). The adipocytes from the mice that had an HFD with HZ supplement were lower in diameter than those of the HFD group (Fig. 4b and c), which suggests that $\mathrm{HZ}$ extract lowers lipid deposition in the mice.

\section{Hyperlipidemia was prevented after $\mathrm{HZ}$ treatment}

Alteration of the lipid composition of serum is one of the signs of metabolic problems [26] and plasma lipid levels were monitored after the diet to evaluate the degree of metabolic deficiency. TG, TC, HDL-C, and LDL$\mathrm{C}$ were measured. Significantly higher levels of TG, TC, 


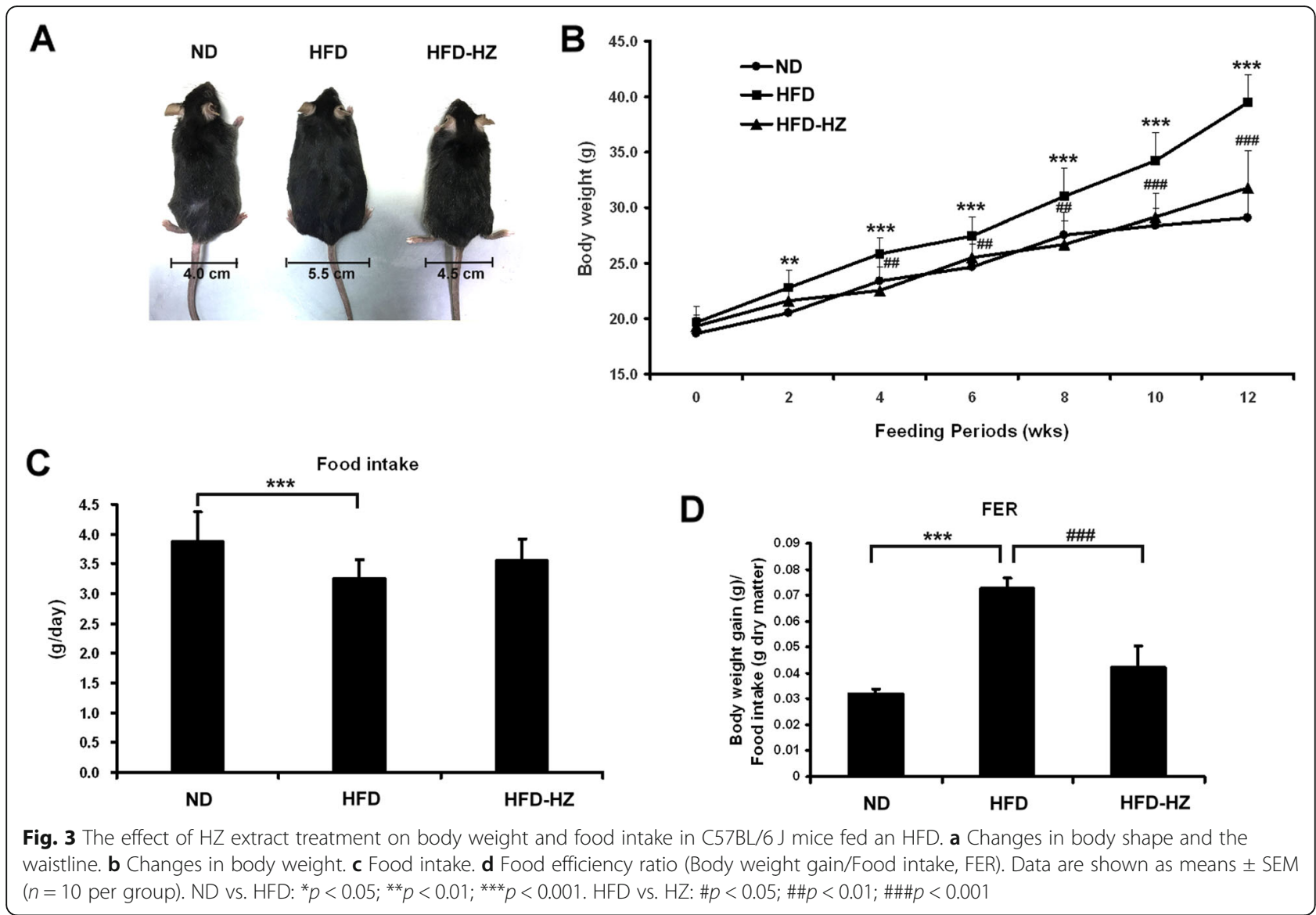

and LDL-C were expressed in the HFD group than the ND group (Fig. 5a, b, and d). Interestingly, the plasma TG, TC, and LDL-C levels in HFD-HZ group were significantly lower than the HFD group. A high HDL-C level was detected in both the HFD and HFD-HZ groups and may be a consequence of the high cholesterol diet. It is suggested that the presence of hypertriglyceridemia and the high cholesterol phenomenon in the HFD mouse model is consistent with the symptom of obesity in humans, which suggests the HZ extract has the potential to inhibit hyperlipidemia bioactivity.

\section{The incidence of steatosis under high-fat conditions was lowered by $\mathrm{HZ}$}

Non-alcoholic fatty liver disease (NAFLD) is one of the criteria for the development of metabolic syndrome, which is mainly due to triglyceride accumulation in the hepatocytes $[27,28]$. To examine the effect of $\mathrm{HZ}$ on fatty liver, the liver was weighed and markers of hepaticsteatosis were evaluated to determine the incidence of steatohepatitis. The livers in the HFD group were heavier than the ND group, but lower when the mice had an HFD diet with the HZ extract (Fig. 6a). The increasing weight of the liver may be attributable to lipid accumulation. In addition, the hepatocytes of the HFD mice became swollen with foaming morphology, showing a lack of staining by $H \& E$ and suggesting more lipid deposition (Fig. 6b). The cell morphology with H\&E staining in the HFD-HZ mouse model was more similar to the ND group. The levels of plasma GOT and GPT help diagnose injury to hepatic tissue $[29,30]$. These markers of hepatic injury, GOT and GPT, were upregulated in HFD mice but maintained at lower levels by treatment with the HZ extract (Fig. 6c and d). There were no significant effects on the pancreas inflammatory marker, LIP (Fig. 6e). These data suggest that HZ extract specifically prevented diet-induced hepatic steatosis and inflammatory response in the liver.

\section{$\mathrm{HZ}$ rescued HFD-induced insulin resistance syndrome}

Previous studies have confirmed the association between dietary fat intake and deteriorated insulin function [31]. The fasting-glucose and insulin levels in HFD mice with or without HFD or HZ extract were measured, reflecting the incidence of insulin resistance syndrome. High fasting blood glucose levels in the HFD group suggested an abnormality of insulin function, while this was prevented by $\mathrm{HZ}$ extract treatment (Fig. 7a). The uncontrolled high-fasting insulin level is considered as the earliest sign of the onset of metabolic syndrome [32]. 


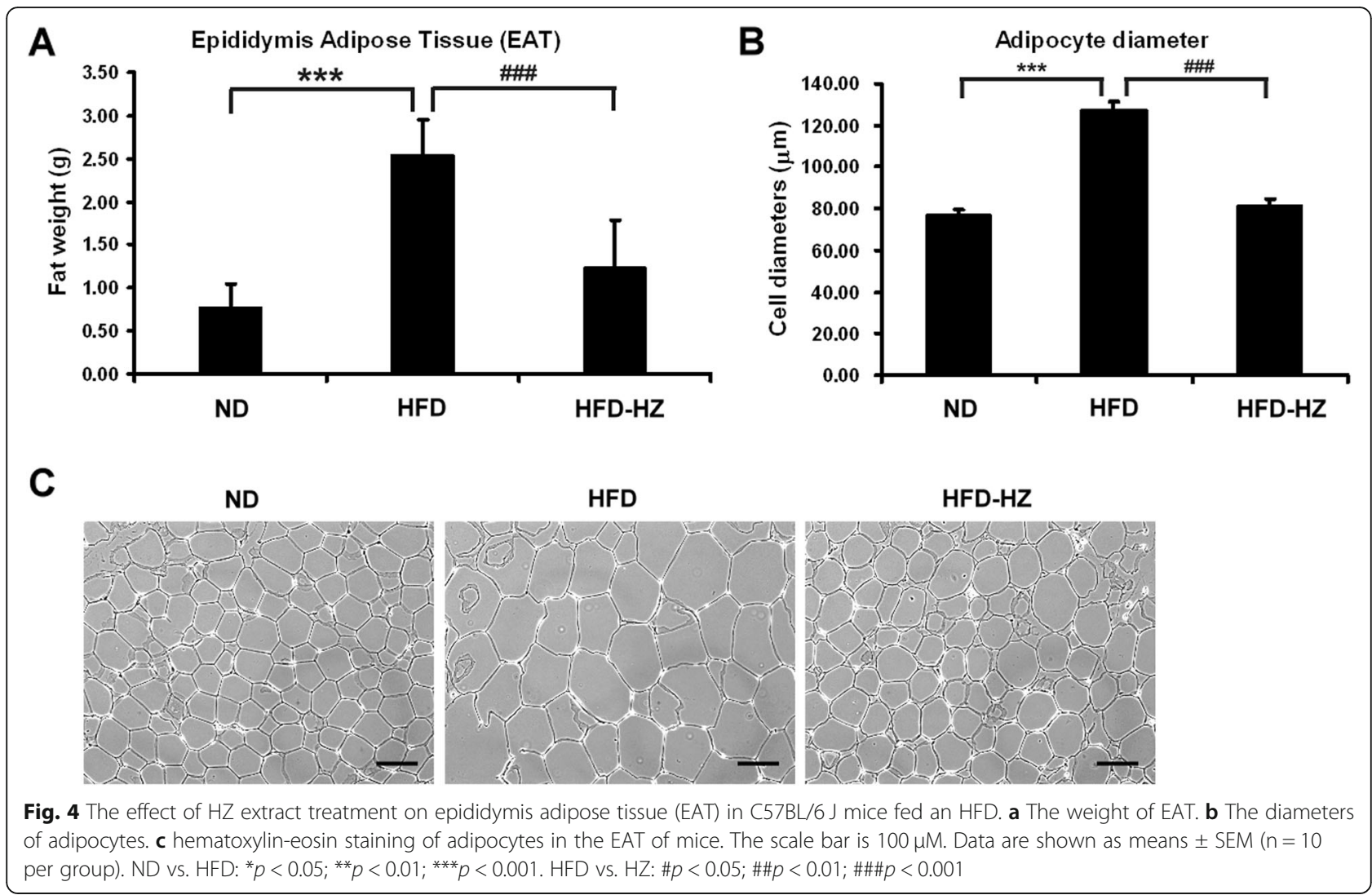

Interestingly, the increase in insulin levels was prevented in the HFD-HZ mice, compared to the HFD group (Fig. 7ba). Generally, insulin resistance is monitored using the homeostasis model assessment of insulin resistance (HOMA-IR) [33]. Therefore, the high HOMA-IR index reflected the increased incidence of diabetes symptoms in HFD mice with high fasting blood glucose and insulin levels. The HOMA-IR index was calculated and found to be at the control level with $\mathrm{HZ}$ extract treatment (Fig. 7c), indicating inhibition of insulin resistance.

\section{Discussion}

Metabolic syndrome associated with insulin resistance has been recognized as a prevalent health issue for decades and has become a burden to many health care systems [34]. Moreover, NAFLD is considered to be closely associated with obesity and metabolic syndrome [35]. Herbal medicines have been shown to have potential therapeutic effects in regulating blood glucose, blood lipid and weight, which are indices for evaluating metabolic syndrome [36]. In our study, HZ extract was prepared, analyzed and its bioactivity examined in regulating an HFD-induced metabolic disorder. The major components of $\mathrm{HZ}$ extract were found to be ugonin $\mathrm{J}$ and ugonin $\mathrm{K}$ (Fig. 1). Consistent with previous findings, $\mathrm{HZ}$ extract was predominantly composed of cyclized geranylflavonoids [37]. Our results showed that when HFD mice were treated with an HZ extract, they were protected against HFD-induced metabolic syndrome.

AMPK is well known to be one of the proteins that regulate metabolic pathways [38]. AMPK activation may protect the liver from lipid accumulation, insulin resistance, and glucose tolerance induced by HFD [39]. Previous studies also showed hepatic activation through the phosphorylation of AMPK suppresses fatty acid synthesis [40]. Our in vitro study showed that $\mathrm{HZ}$ extract significantly increased the phosphorylation of AMPK and the phosphorylation of AMPK's downstream enzyme ACC. This suggests HZ extract facilitated AMPK and ACC activation in cells under high-fat conditions, and reduced palmitate-induced cellular lipid accumulation in hepatocytes.

Generally, an HFD promotes severe changes, such as hepatic steatosis, $\beta$ oxidation status and the balance of oxidants, which has effects on body weight, insulin signaling and other metabolic parameters [41]. HFD mice have abnormalities in lipid and glucose metabolism. Here, with the human fatty liver cell model and the HFD mice model, we aimed to demonstrate the effects of the $\mathrm{HZ}$ extract on the pathogenesis of liver-related metabolic syndrome and dyslipidemia. In terms of protecting the liver from oxidative stress and inflammation, we 

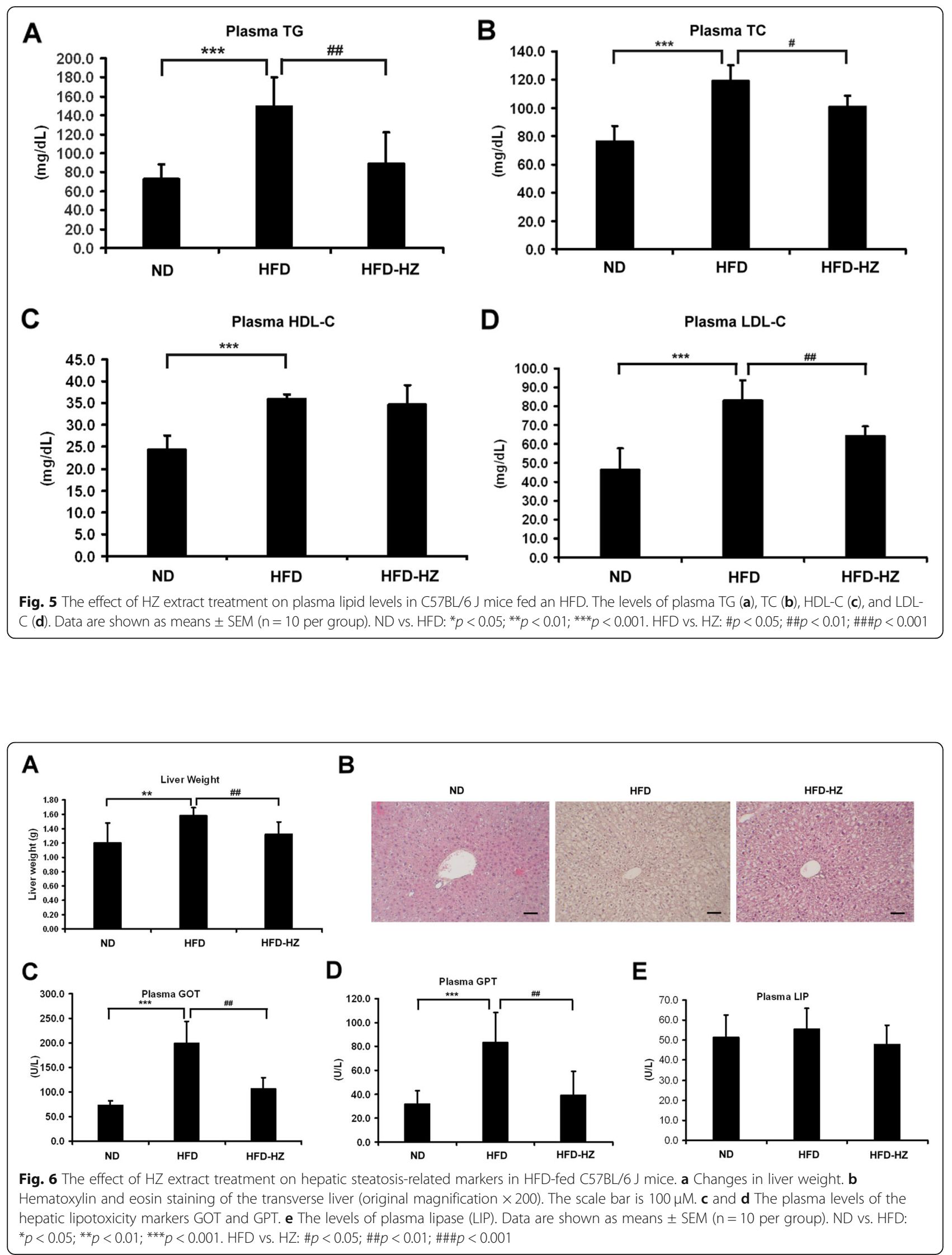


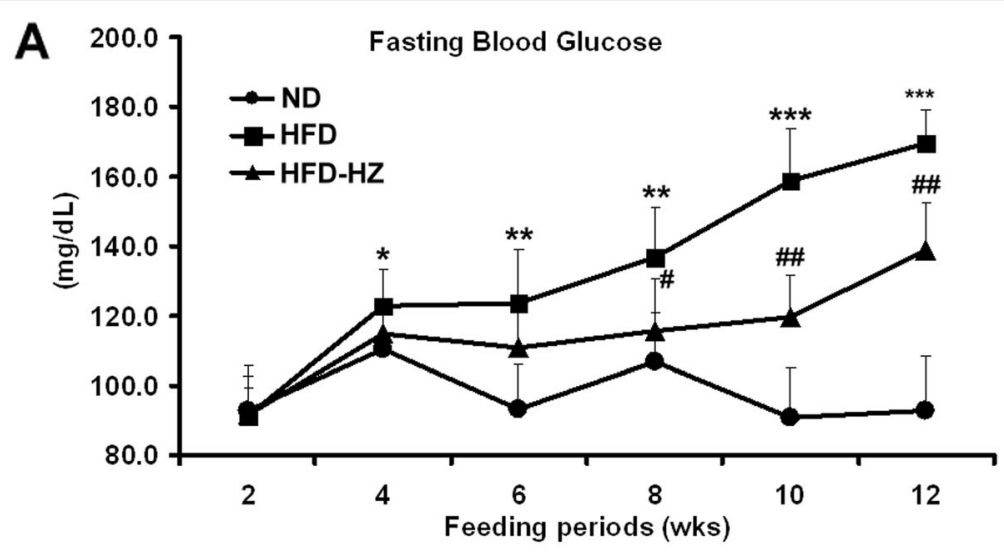

B
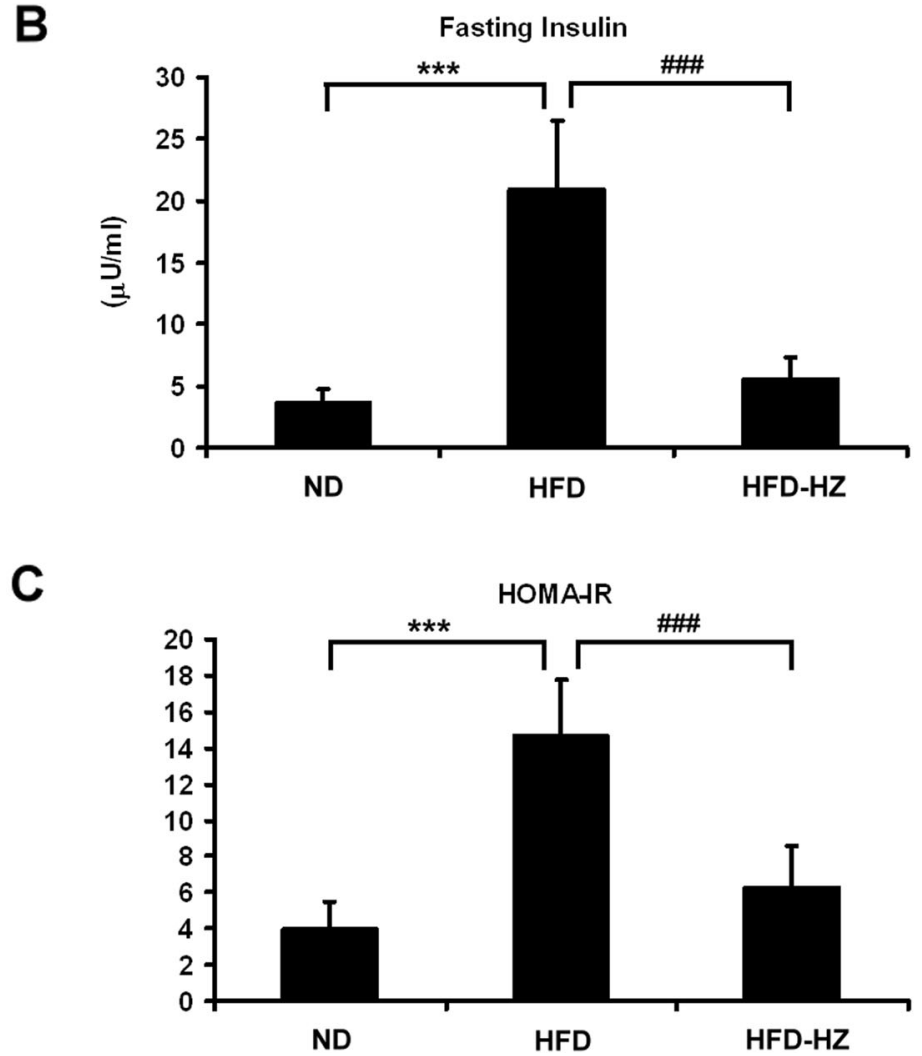

Fig. 7 The effect of $\mathrm{HZ}$ extract treatment on blood glucose and insulin resistance in HFD-fed C57BL/6 J mice. a Levels of blood glucose after $12 \mathrm{~h}$ of fasting. $\mathbf{b}$ Levels of plasma insulin after $12 \mathrm{~h}$ of fasting. $\mathbf{c}$ The HOMA-IR index calculated using fasting blood glucose and insulin levels. Data are

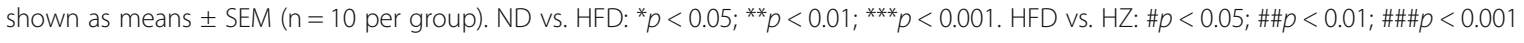

confirmed the effects of $\mathrm{HZ}$ extract on regulating lipid metabolism. However, the pharmacokinetics of HZ extract remains unclear and needs further clarification.

Previously, twelve flavonoids were identified in ethanolextracted HZ through HPLC and NMR analysis [11]. Among the flavonoids in HZ, many bioactivities have been demonstrated, such as antioxidative activity [11], antiinflammatory function [37], antiosteoporosis [12], and anticancer [42, 43] and hepatoprotective effects [44]. In addition, plant-derived flavondoids recently were proposed as a health supplement [45]. For example, tea-extracted catechins and theaflavins improved plasma lipid absorption [46] and have been used for hyperlipidemia therapy. Furthermore, solid evidence proved that cinnamon extract regulated the metabolism of carbohydrates and lipids through peroxisome proliferator-activated receptors (PPARs) in obesity and diabetic mouse models [47, 48]. Moreover, anthocyanin C3G, a flavonoid, inhibited excessive ROS production by activating GSH synthesis, which many consider to be controllers of hyperglycemia-induced 
hepatic oxidative damage [49]. Based on this evidence of the therapeutic potential of flavonoids, the $\mathrm{HZ}$ extract was shown to be effective in restoring metabolic syndrome induced by an HFD. However, the detailed mechanisms of action of ugonins $\mathrm{J}$ and $\mathrm{K}$, and perhaps other bioactive compounds in $\mathrm{HZ}$, require to be elucidated.

\section{Conclusions}

Our results showed that HZ extract prevented increases in body weight and fat accumulation around the waist in mice fed a 12-week-HFD diet. Food intake efficiency was decreased along, with a reduction in the accumulation of fat that may cause metabolic syndrome. HZ treatment also inhibited the extremely high level of bad cholesterol transportation carriers, LDL-C, in HFD mice, these being considered a sign of health problems. The protective effect on metabolism was examined in terms of concentrations of glucose, lipids, and other insulin resistanceassociated index. Further investigation of the molecular mechanism showed that $\mathrm{HZ}$ extract upregulated the genes and proteins associated with fatty acids oxidation, downregulated those related to hepatic de novo lipogenesis in palmitate-treated human HuS-E/2 hepatocytes. These solid results indicate that HZ has a promising bioactivity in regulating obesity and insulin sensitivity, which may have potential for clinical application in preventing from hepatic steatosis and insulin resistance.

\section{Supplementary information}

Supplementary information accompanies this paper at https://doi.org/10. 1186/s12906-019-2782-3.

Additional file 1: Table S1. Primer sequences in RT-PCR

\begin{abstract}
Abbreviations
ACC: Acetyl-CoA carboxylase; AMPK: AMP-activated protein kinase; CPT1: Carnitine palmitoyltransferase I; EAT: Epididymis adipose tissue; FER: Food efficiency ratios; GOT: Glutamic oxaloacetic transaminase; GPT: Glutamic pyruvic transaminase; HDL-C: HDL-cholesterol; HFD: High fat diet; HOMA-IR: Homeostasis model assessment of insulin resistance; HZ: Helminthostachys zeylanica; LDL-C: Low-density lipoprotein cholesterol; LIP: Lipase; NAFLD: Nonalcoholic fatty liver disease; ND: normal diet; PPARa: Peroxisome proliferator-activated receptor alpha; PPARy: Peroxisome proliferator-activated receptor gamma; PPARS: Peroxisome proliferatoractivated receptor delta; SREBP-1c: Sterol regulatory element-binding transcription factor 1C; T2DM: Type 2 diabetes mellitus; TC: Total cholesterol; TG: Triglyceride
\end{abstract}

\section{Acknowledgements}

We thank Dr. K. Shimotohno (Kyoto University, Japan) for providing HuS-E/2 cells.

\section{Authors' contributions}

TCC, HC, HCH, YLH and YHL carried out the experiments. YCL, HKL and $\mathrm{CH}$ analyzed the data. TCC and $\mathrm{CH}$ wrote the paper. All authors read and approved the final manuscript.

\section{Funding}

This work was supported by research grant MOST 104-2320-B-077-003- and MOST 106-2320-B-010-038- from the Ministry of Science and Technology, Taiwan. The funders had no role in study design; in the collection, analysis and interpretation of data; in the writing of the articles; and in the decision to submit it for publication.

\section{Availability of data and materials}

The datasets used and/or analyzed during the current study available from the corresponding author on reasonable request.

Ethics approval and consent to participate

All animal experiments, including mouse manipulations (e.g. dosing and bleeding), were performed under a protocol (IACUC permit number 105520-214-01-637) approved by the Animal Research Committee of the NRICM (Taipei, Taiwan)

Consent for publication

Not applicable.

\section{Competing interests}

The authors declare that they have no competing interests.

\section{Author details}

${ }^{1}$ Ph.D. Program in Medical Biotechnology, College of Medical Science and Technology, Taipei Medical University, Taipei 11001, Taiwan. ${ }^{2}$ Department of Biotechnology and Laboratory Science in Medicine, National Yang-Ming University, No. 155, Sec. 2, Linong St., Beitou District, Taipei 11221, Taiwan. ${ }^{3}$ Department of Chemistry, Chinese Culture University, Taipei 11114, Taiwan. ${ }^{4}$ National Research Institute of Chinese Medicine, Ministry of Health and Welfare, Taipei 11221, Taiwan. ${ }^{5}$ Department of Cosmetic Science, Chang Gung University of Science and Technology, Taoyuan, Taiwan. ${ }^{6}$ Department of Applied Science, National Tsing Hua University, Hsinchu 30014, Taiwan. ${ }^{7}$ School of Medical Laboratory Science and Biotechnology, College of Medical Science and Technology, Taipei Medical University, Taipei 11001, Taiwan. ${ }^{8}$ Ph.D. Program in Clinical Drug Development of Chinese Herbal Medicine, Taipei Medical University, Taipei 11001, Taiwan. ${ }^{9}$ Department of Earth and Life Sciences, University of Taipei, Taipei 11153, Taiwan.

Received: 11 September 2019 Accepted: 29 November 2019

Published online: 13 December 2019

\section{References}

1. Dunn W, Xu R, Wingard DL, Rogers C, Angulo P, Younossi ZM, Schwimmer $J B$. Suspected nonalcoholic fatty liver disease and mortality risk in a population-based cohort study. Am J Gastroenterol. 2008;103(9):2263-71.

2. Postic C, Girard J. Contribution of de novo fatty acid synthesis to hepatic steatosis and insulin resistance: lessons from genetically engineered mice. J Clin Invest. 2008;118(3):829-38.

3. Masuoka HC, Chalasani N. Nonalcoholic fatty liver disease: an emerging threat to obese and diabetic individuals. Ann N Y Acad Sci. 2013;1281:106-22.

4. Parekh S, Anania FA. Abnormal lipid and glucose metabolism in obesity: implications for nonalcoholic fatty liver disease. Gastroenterology. 2007: 132(6):2191-207

5. Marchesini G, Brizi M, Morselli-Labate AM, Bianchi G, Bugianesi E, McCullough AJ, Forlani G, Melchionda N. Association of nonalcoholic fatty liver disease with insulin resistance. Am J Med. 1999;107(5):450-5.

6. Adams LA, Angulo P. Treatment of non-alcoholic fatty liver disease. Postgrad Med J. 2006;82(967):315-22.

7. Nobili V, Manco M, Devito R, Di Ciommo V, Comparcola D, Sartorelli MR, Piemonte F, Marcellini M, Angulo P. Lifestyle intervention and antioxidant therapy in children with nonalcoholic fatty liver disease: a randomized, controlled trial. Hepatology. 2008;48(1):119-28.

8. Kitade H, Chen G, Ni Y, Ota T. Nonalcoholic Fatty Liver Disease and Insulin Resistance: New Insights and Potential New Treatments. Nutrients. 2017;9(4).

9. Su LH, Li YP, Li HM, Dai WF, Liu D, Cao L, Li RT. Anti-inflammatory Prenylated flavonoids from Helminthostachys zeylanica. Chem Pharm Bull (Tokyo). 2016;64(5):497-501.

10. Huang YC, Hwang TL, Yang YL, Wu SH, Hsu MH, Wang JP, Chen SC, Huang LJ, Liaw CC. Acetogenin and prenylated flavonoids from Helminthostachys zeylanica with inhibitory activity on superoxide generation and elastase release by neutrophils. Planta Med. 2010;76(5):447-53.

11. Huang YL, Yeh PY, Shen CC, Chen CC. Antioxidant flavonoids from the rhizomes of Helminthostachys zeylanica. Phytochemistry. 2003;64(7):1277-83. 
12. Lee $\mathrm{CH}$, Huang YL, Liao JF, Chiou WF. Ugonin K-stimulated osteogenesis involves estrogen receptor-dependent activation of non-classical Src signaling pathway and classical pathway. Eur J Pharmacol. 2012;676(1-3): 26-33.

13. Yang CM, Yang SH, Lee TH, Fang JY, Lin CF, Jou MJ, Hsieh HL. Evaluation of anti-inflammatory effects of Helminthostachys zeylanica extracts via inhibiting Bradykinin-induced MMP-9 expression in brain astrocytes. Mol Neurobiol. 2016;53(9):5995-6005.

14. Pan CH, Li PC, Chien YC, Yeh WT, Liaw CC, Sheu MJ, Wu CH. Suppressive activities and mechanisms of ugonin $\mathrm{J}$ on vascular smooth muscle cells and balloon angioplasty-induced neointimal hyperplasia. Phytother Res. 2018; 32(2):312-20.

15. Suja SR, Latha PG, Pushpangadan P, Rajasekharan S. Evaluation of hepatoprotective effects of Helminthostachys zeylanica (L.) hook against carbon tetrachloride-induced liver damage in Wistar rats. J Ethnopharmacol. 2004;92(1):61-6.

16. Liu HK, Hung TM, Huang HC, Lee IJ, Chang CC, Cheng JJ, Lin LC, Huang C. Bai-Hu-Jia-Ren-Shen-tang decoction reduces fatty liver by activating AMPactivated protein kinase in vitro and in vivo. Evid Based Complement Alternat Med. 2015;2015:651734.

17. Anstee QM, Goldin RD. Mouse models in non-alcoholic fatty liver disease and steatohepatitis research. Int J Exp Pathol. 2006;87(1):1-16.

18. Aly HH, Watashi K, Hijikata M, Kaneko H, Takada Y, Egawa H, Uemoto S, Shimotohno K. Serum-derived hepatitis $C$ virus infectivity in interferon regulatory factor-7-suppressed human primary hepatocytes. J Hepatol. 2007; 46(1):26-36

19. Huang HC, Tao MH, Hung TM, Chen JC, Lin ZJ, Huang C. Epigallocatechin-3gallate inhibits entry of hepatitis B virus into hepatocytes. Antivir Res. 2014; 111:100-11.

20. Chiang H, Lee JC, Huang HC, Huang H, Liu HK, Huang C. Delayed intervention with a novel SGLT2 inhibitor NGl001 suppresses diet-induced metabolic dysfunction and non-alcoholic fatty liver disease in mice. $\mathrm{Br}$ Pharmacol. 2019

21. Zambo V, Simon-Szabo L, Szelenyi P, Kereszturi E, Banhegyi G, Csala M. Lipotoxicity in the liver. World J Hepatol. 2013;5(10):550-7.

22. Hardie DG. AMP-activated/SNF1 protein kinases: conserved guardians of cellular energy. Nat Rev Mol Cell Biol. 2007;8(10):774-85.

23. Yamauchi K, Mitsunaga T, Itakura Y, Batubara I. Extracellular melanogenesis inhibitory activity and the structure-activity relationships of ugonins from Helminthostachys zeylanica roots. Fitoterapia. 2015;104:69-74.

24. Kwon EY, Jung UJ, Park T, Yun JW, Choi MS. Luteolin attenuates hepatic steatosis and insulin resistance through the interplay between the liver and adipose tissue in mice with diet-induced obesity. Diabetes. 2015;64(5):1658-69.

25. Lopez-Vinas E, Bentebibel A, Gurunathan C, Morillas M, de Arriaga D, Serra D, Asins G, Hegardt FG, Gomez-Puertas P. Definition by functional and structural analysis of two malonyl-CoA sites in carnitine palmitoyltransferase 1A. J Biol Chem. 2007:282(25):18212-24.

26. Canals M, Figueroa D, Alfaro C, Kawamoto T, Torres-Contreras H, Sabat P, Veloso C. Effects of diet and water supply on energy intake and water loss in a mygalomorph spider in a fluctuating environment of the Central Andes. J Insect Physiol. 2011;57(11):1489-94.

27. Williams T. Metabolic syndrome: nonalcoholic fatty liver disease. FP Essent. 2015;435:24-9.

28. Asrih M, Jornayvaz FR. Metabolic syndrome and nonalcoholic fatty liver disease: is insulin resistance the link? Mol Cell Endocrinol. 2015:418(Pt 1):55-65.

29. Cassidy WM, Reynolds TB. Serum lactic dehydrogenase in the differential diagnosis of acute hepatocellular injury. J Clin Gastroenterol. 1994;19(2):118-21.

30. Dufour DR, Lott JA, Nolte FS, Gretch DR, Koff RS, Seeff LB. Diagnosis and monitoring of hepatic injury. II. Recommendations for use of laboratory tests in screening, diagnosis, and monitoring. Clin Chem. 2000;46(12):2050-68.

31. Huang T, Beaty T, Li J, Liu H, Zhao W, Wang Y. Association between dietary fat intake and insulin resistance in Chinese child twins. Br J Nutr. 2017 $117(2): 230-6$.

32. Ling JC, Mohamed MN, Jalaludin MY, Rampal S, Zaharan NL, Mohamed Z. Determinants of high fasting insulin and insulin resistance among overweight/obese adolescents. Sci Rep. 2016;6:36270.

33. Morimoto A, Tatsumi Y, Soyano F, Miyamatsu N, Sonoda N, Godai K, Ohno $Y$, Noda M, Deura K. Increase in homeostasis model assessment of insulin resistance (HOMA-IR) had a strong impact on the development of type 2 diabetes in Japanese individuals with impaired insulin secretion: the Saku study. PLoS One. 2014;9(8):e105827.
34. Yin J, Zhang H, Ye J. Traditional chinese medicine in treatment of metabolic syndrome. Endocr Metab Immune Disord Drug Targets. 2008;8(2):99-111.

35. Haas JT, Francque S, Staels B. Pathophysiology and mechanisms of nonalcoholic fatty liver disease. Annu Rev Physiol. 2016:78:181-205.

36. Jang S, Jang BH, Ko Y, Sasaki Y, Park JS, Hwang EH, Song YK, Shin YC, Ko SG. Herbal medicines for treating metabolic syndrome: a systematic review of randomized controlled trials. Evid Based Complement Alternat Med. 2016; 2016:5936402.

37. Huang YC, Hwang TL, Chang CS, Yang YL, Shen CN, Liao WY, Chen SC, Liaw CC. Anti-inflammatory flavonoids from the rhizomes of Helminthostachys zeylanica. J Nat Prod. 2009;72(7):1273-8.

38. Hardie DG. AMPK: a key regulator of energy balance in the single cell and the whole organism. Int J Obes. 2008;32(S4):S7-S12.

39. Higuchi N, Kato M, Tanaka M, Miyazaki M, Takao S, Kohjima M, Kotoh K, Enjoji M, Nakamuta M, Takayanagi R. Effects of insulin resistance and hepatic lipid accumulation on hepatic mRNA expression levels of apoB, MTP and LFABP in non-alcoholic fatty liver disease. Experimental and Therapeutic Medicine. 2011;2(6):1077-81.

40. Foretz M, Viollet B. Regulation of hepatic metabolism by AMPK. J Hepatol. 2011;54(4):827-9.

41. Kakimoto PA, Kowaltowski AJ. Effects of high fat diets on rodent liver bioenergetics and oxidative imbalance. Redox Biol. 2016;8:216-25.

42. Nandakumar V, Singh T, Katiyar SK. Multi-targeted prevention and therapy of cancer by proanthocyanidins. Cancer Lett. 2008:269(2):378-87.

43. Dai Q, Shu XO, Jin F, Potter JD, Kushi LH, Teas J, Gao YT, Zheng W. Population-based case-control study of soyfood intake and breast cancer risk in Shanghai. Br J Cancer. 2001;85(3):372-8.

44. Wu KC, Ho YL, Kuo YH, Huang SS, Huang GJ, Chang YS. Hepatoprotective Effect of Ugonin M, A Helminthostachyszeylanica Constituent, on Acetaminophen-Induced Acute Liver Injury in Mice. Mol. 2018;23(10).

45. Yetley EA. Multivitamin and multimineral dietary supplements: definitions, characterization, bioavailability, and drug interactions. Am J Clin Nutr. 2007; 85(1):269S-76S.

46. McGowan MP, Proulx S. Nutritional supplements and serum lipids: does anything work? Curr Atheroscler Rep. 2009;11(6):470-6.

47. Sheng X, Zhang Y, Gong Z, Huang C, Zang YQ. Improved insulin resistance and lipid metabolism by cinnamon extract through activation of peroxisome proliferator-activated receptors. PPAR Res. 2008;2008:581348.

48. Qin B, Polansky MM, Anderson RA. Cinnamon extract regulates plasma levels of adipose-derived factors and expression of multiple genes related to carbohydrate metabolism and lipogenesis in adipose tissue of fructosefed rats. Horm Metab Res. 2010;42(3):187-93.

49. Zhu W, Jia $Q$, Wang $Y$, Zhang $Y$, Xia $M$. The anthocyanin cyanidin-3-O- $\beta$ glucoside, a flavonoid, increases hepatic glutathione synthesis and protects hepatocytes against reactive oxygen species during hyperglycemia: involvement of a CAMP-PKA-dependent signaling pathway. Free Radic Biol Med. 2012;52(2):314-27.

\section{Publisher's Note}

Springer Nature remains neutral with regard to jurisdictional claims in published maps and institutional affiliations.

Ready to submit your research? Choose BMC and benefit from:

- fast, convenient online submission

- thorough peer review by experienced researchers in your field

- rapid publication on acceptance

- support for research data, including large and complex data types

- gold Open Access which fosters wider collaboration and increased citations

- maximum visibility for your research: over $100 \mathrm{M}$ website views per year

At $\mathrm{BMC}$, research is always in progress.

Learn more biomedcentral.com/submission 